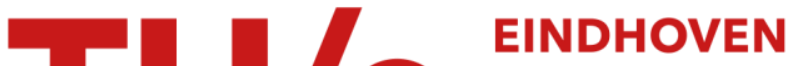 UNIVERSITY OF TECHNOLOGY
}

\section{Modelling the temperature evolution of bone under high intensity focused ultrasound}

\section{Citation for published version (APA):}

ten Eikelder, H. M. M., Bosnacki, D., Elevelt, A., Donato, K., Di Tullio, A., Breuer, B. J. T., van Wijk, J. H., van Dijk, E. V. M., Modena, D., Yeo, S. Y., \& Grull, H. (2016). Modelling the temperature evolution of bone under high intensity focused ultrasound. Physics in Medicine and Biology, 61(4), 1810-1828.

https://doi.org/10.1088/0031-9155/61/4/1810

DOI:

10.1088/0031-9155/61/4/1810

Document status and date:

Published: 21/02/2016

\section{Document Version:}

Accepted manuscript including changes made at the peer-review stage

\section{Please check the document version of this publication:}

- A submitted manuscript is the version of the article upon submission and before peer-review. There can be important differences between the submitted version and the official published version of record. People interested in the research are advised to contact the author for the final version of the publication, or visit the $\mathrm{DOI}$ to the publisher's website.

- The final author version and the galley proof are versions of the publication after peer review.

- The final published version features the final layout of the paper including the volume, issue and page numbers.

Link to publication

\section{General rights}

Copyright and moral rights for the publications made accessible in the public portal are retained by the authors and/or other copyright owners and it is a condition of accessing publications that users recognise and abide by the legal requirements associated with these rights.

- Users may download and print one copy of any publication from the public portal for the purpose of private study or research.

- You may not further distribute the material or use it for any profit-making activity or commercial gain

- You may freely distribute the URL identifying the publication in the public portal.

If the publication is distributed under the terms of Article 25fa of the Dutch Copyright Act, indicated by the "Taverne" license above, please follow below link for the End User Agreement:

www.tue.nl/taverne

Take down policy

If you believe that this document breaches copyright please contact us at:

openaccess@tue.nl

providing details and we will investigate your claim. 


\title{
Modeling the temperature evolution of bone under High Intensity Focused Ultrasound
}

\author{
H M M ten Eikelder ${ }^{1}$, D Bošnački ${ }^{1}$, A Elevelt ${ }^{2}, \mathrm{~K}$ Donato $^{2}, \mathrm{~A}$ \\ Di Tullio ${ }^{2}$, B J T Breuer ${ }^{1}$, J H van Wijk ${ }^{1}, \mathbf{E}$ V M van Dijk ${ }^{1}$, D \\ Modena $^{1}$, S Y Yeo ${ }^{1}$ and H Grüll ${ }^{1,2}$ \\ ${ }^{1}$ Eindhoven University of Technology, Eindhoven, The Netherlands \\ ${ }^{2}$ Department of Minimally Invasive Healthcare, Philips Research Eindhoven, the \\ Netherlands \\ E-mail: h.m.m.ten.eikelder@tue.nl, katia.donato@philips.com
}

December 2015

\begin{abstract}
Magnetic Resonance-guided High Intensity Focused Ultrasound (MRHIFU) has been clinically shown to be effective for palliative pain management in patients suffering from skeletal metastasis. The underlying mechanism is supposed to be periosteal denervation caused by ablative temperatures reached through ultrasound heating of the cortex. Challenge is exact temperature control during sonication as MR-based thermometry approaches for bone tissue are currently not available. Thus, in contrast to MR-HIFU ablation of soft tissue, a thermometry feedback to the HIFU is lacking and treatment of bone metastasis is entirely based on temperature information acquired in soft tissue adjacent to the bone surface. However, heating of the adjacent tissue depends on the exact sonication protocol and requires extensive modeling to estimate the actual temperature of the cortex. Here we develop a computational model to calculate the spatial temperature evolution in bone and adjacent tissue during sonication. First, a ray tracing technique is used to compute the heat production in each spatial point serving as a source term for the second part, where the actual temperature is calculated as function of space and time by solving Pennes bio-heat equation. Importantly, our model includes shear waves that arise at the bone interface as well as all geometrical considerations of transducer and bone geometry. The model was compared with a theoretical approach based on the far field approximation and an MR-HIFU experiment using a bone phantom. Furthermore we investigated the contribution of shear waves to the heat production and resulting temperatures in bone. The temperature evolution predicted by our model was in accordance with the far field approximation and agreed well with the experimental data obtained in phantoms. Our model allows simulating HIFU treatments of bone metastasis in patients and can be extended to a planning tool prior to MR-HIFU treatments.
\end{abstract}

PACS numbers: 87.50.yt, 43.80.Gx, 43.80.Sh, 42.15.Dp

Keywords: high intensity focussed ultrasound, thermal ablation, bone, ray tracing. Submitted to: Phys. Med. Biol. 


\section{Introduction}

Bone is the most common target site for the formation of distant metastases originating from solid tumors such as breast or prostate cancer (Weilbaecher et al. 2011). At this stage, the disease is virtually incurable with limited life expectancy and reduced quality of life (Coleman 2006). Treatment is palliative and directed to alleviate pain often associated with bone metastases. Treatment options in the management of bone metastases comprise systemic therapies such as chemotherapy, hormonal therapy, or bone seeking bisphosphonates, as well as local treatments such as surgery, radiation therapy, and thermal ablation. Various thermal ablation techniques exist, like radiofrequency, microwave, laser or High Intensity Focused Ultrasound (Kurup \& Callstrom 2010, Filippiadis et al. 2014). High Intensity Focused Ultrasound (HIFU) has been used with promising outcomes (Hurwitz 2014, Huisman et al. 2014, Napoli et al. 2013a, Napoli et al. 2013b, Huisman \& van den Bosch 2011, Li et al. 2010, Chen et al. 2010, Liberman et al. 2009). HIFU is a non-invasive method that allows rapid heating of subcutaneous tissues to ablative temperatures to induce coagulative necrosis (Haar \& Coussios 2007, Hynynen 2010). This method is now clinically used for thermal ablation of uterine fibroids and palliative treatment of bone metastasis with other applications being under investigation (Aubry et al. 2013, Foley et al. 2013). HIFU treatment is usually being performed image-guided using either diagnostic ultrasound or Magnetic Resonance Imaging(MRI) for treatment planning and spatial guidance. MR-guided HIFU (MR-HIFU) has the additional benefit that MRI can obtain near-real time temperature information of the heated tissue via proton resonance frequency shift (PRFS) thermometry (Denis De Senneville et al. 2005, Köhler et al. 2009, McDannold 2005, Rieke \& Butts Pauly 2008). The temperature map can be used to achieve a closed-loop feedback to the HIFU in order to obtain and maintain a well-defined temperature. In thermal ablation of water-rich soft tissue, such as uterine fibroids, the PRFS method works well as the transversal relaxation time (T2) of this water-rich tissue is sufficiently long. Current PRFS-based temperature mapping fails in bone tissue due to too short T2 and upmost provides a temperature map for the adjacent soft tissue but not for the cortex itself, though new MR-based thermometry techniques for bone are currently under investigation (Davis \& Warren 2014, Ramsay et al. 2014). Controlled heating of the bone with HIFU is furthermore complicated as soft tissue and the bone cortex strongly differ in their acoustic and thermal properties (Goss et al. 1979, Nell \& Meyers 2010). Bone has a much higher absorption of acoustic energy but a lower thermal conductivity compared to soft tissue. Furthermore, the soft tissue-bone interface gives rise to reflected, transmitted ultrasound waves as well as shear waves propagating inside the cortex but with little penetration depth (Pinton et al. 2012). Applying HIFU to the bone will therefore lead to fast heating of the cortex which subsequently serves as a heat source that will indirectly lead to ablation of the adjacent soft tissue such as the periosteum, which is considered to be the most prominent source of pain at least in

osteoblastic lesions. Thus, understanding the exact interaction of HIFU with bone and 
predicting the temperature elevation in bone and surrounding tissue as a result of this interaction is required to design improved treatment protocols.

To date, different studies have been performed investigating the effects of ultrasound on bone, assessing the thermal damage at bone-tissue interface, and addressing safety issues for soft tissue around bone using a modeling approach (Fujii et al. 1999, Lu et al. 2000, Lin et al. 2000, Myers 2004, Nell \& Meyers 2010, Moros et al. 1999, Moros et al. 2000, Scott et al. 2013b, Scott et al. 2014). However, often these models assume the muscle-bone interface to be planar, shear waves are not taken into account, other transducer geometries are used (e.g. flat transducers), or interstitial ultrasound ablation is considered. Here, we describe a simple approach to compute the temperature in bone and soft tissue due to HIFU treatment. The acoustic power flow (acoustic intensity) of the HIFU transducer is computed using a ray tracer approach, which is used to calculate the heat deposition per volume. The ray tracer model takes both longitudinal waves and shear waves, the latter for solids only, into account. Subsequently, temperature distributions are calculated by solving the Pennes bio-heat equation (Pennes 1948) using the heat deposition computed by the ray tracer as source term. We use a realistic 3d transducer geometry and allow cylindrically shaped bones (including marrow), that can also be tilted with respect to the US beam. We compared the heat production calculated by the ray tracer with the heat production computed using the far field approximation for the pressure field and made a detailed study of the contribution of shear waves to the heat production. The temperature profiles computed by our method were also compared with first results of an experiment using a gel-bone phantom setup, where the temperature was measured with MR-thermometry in gel and with sensors in the bone.

\section{Materials and Methods}

\subsection{Modeling approach}

The computation of the temperature evolution of objects under HIFU consists of two parts. First the heat production density (in $\mathrm{W} / \mathrm{cm}^{3}$ ) by the ultrasound waves for each point in the objects has to be computed. This part involves the propagation, attenuation, reflection and refraction of ultrasound waves in different media. Moreover in configurations where solids like bone are present also shear (transversal) waves and the corresponding mode-conversion have to be considered. In the second part the actual temperature evolution is obtained by solving the heat equation that has the computed heat production as a source term. In an in-vivo situation this heat equation has to be replaced by the bio-heat equation, taking also the heat flux by perfusion into account.

2.1.1. Computing the Heat Production Density The model takes the actual geometry of the phased array transducer used in the Philips Sonalleve system into account (Köhler et al. 2009). This phased-array HIFU transducer consists of 256 element placed on a spherical shell (12 cm radius of curvature, $13 \mathrm{~cm}$ aperture) operating at $1.4 \mathrm{MHz}$, 
creating a cigar-shaped focus point with a dimension of ca. $2 \mathrm{~mm}$ width and $7 \mathrm{~mm}$ length. The flow of acoustic power from each transducer element is described by a number of rays, that have as target randomly selected points within a $2 \mathrm{~mm}$ diameter circle in the focal plane, approximating the focal region described above. Each ray has an initial power $P_{\text {tot }} / N_{\text {rays }}$, where $P_{\text {tot }}$ is the total acoustic power and $N_{\text {rays }}(>=100000)$ is the total number of rays starting from all the transducer elements. In one material type rays propagate along straight lines and their power is attenuated using the attenuation coefficient of the considered material. If $I(s)$ is the power flux in a ray after $s \mathrm{~cm}$ in a material with attenuation coefficient (for the pressure) $\alpha$, then, since acoustic power is proportional to the square of the pressure, $I(s)=I(0) e^{-2 \alpha s}$.

Waves propagates differently in liquids and in solids. In liquids only longitudinal (pressure) waves can travel, whereas in solids also shear waves are possible. For our model, muscle and marrow are considered as liquid, with ultrasound properties similar to water. Thus, rays in muscle and marrow always describe longitudinal (pressure) waves. Conversely, cortical bone is considered as a solid. Rays in solids may represent longitudinal or shear waves. In the latter case, besides a power, a shear ray also has a polarization vector, describing the oscillation direction of the particles. At an interface between two material types the incoming ray terminates and new reflected and transmitted (refracted) rays are generated. The directions of these reflected and transmitted rays are determined by Snell's law, the power distribution comes from the acoustic version of the Fresnel relations. At the liquid-solid interface like muscle-bone also mode-conversion takes place. The incoming ray from the liquid gives rise to three new rays: a reflected ray in the liquid, a transmitted (refracted) longitudinal ray in the solid and a transmitted (refracted) shear ray in the solid.

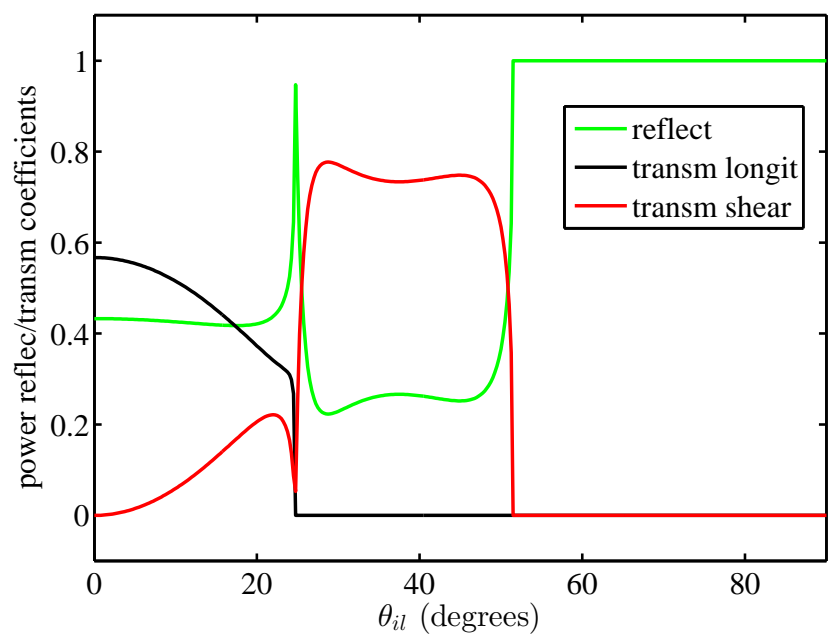

Figure 1. Mode conversion at the muscle-bone interface with the power reflection and transmission coefficients.

Figure 1 shows the power reflection/transmission coefficients at the muscle-bone interface, as calculated from Fresnel relations, see suppl. info and references given 
therein for the computation of the Fresnel Relations. Mode conversion also occurs in the opposite direction, i.e., at a solid-liquid interface like bone-muscle or bone-marrow. Shear waves in the solid may give rise to refracted longitudinal waves in the liquid. This depends also the polarization direction of the shear waves, see suppl. info. An overview of the ray tracing process is shown in Figure 2 .

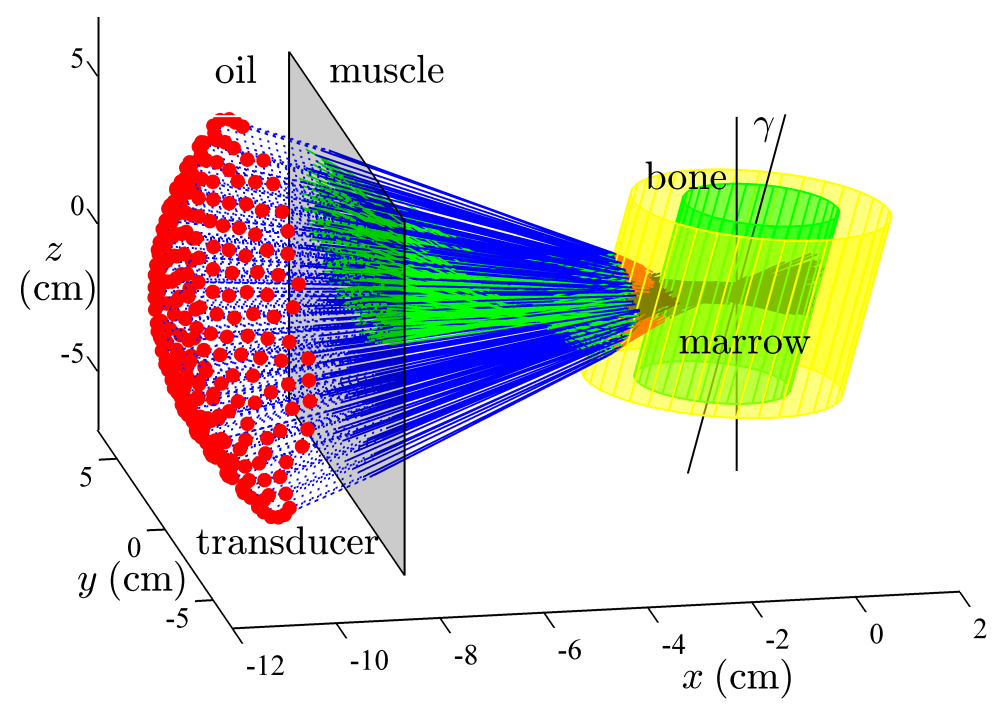

Figure 2. Ray tracer configuration. Color for the outgoing rays from the transducer: blue; after reflection: green; shear rays in bone: red; longitudinal rays in bone or marrow: black. Rays in the (lossless) oil are shown dashed. The angle $\gamma$ gives the inclination in the $x-z$ plane of the bone axis with respect to the $y$ axis. The center of focus is located at $(0,0,0)$.

For computing the generated heat, each ray is divided in small segments of length $\Delta s$. The attenuation of acoustic power in a segment between distances $s$ and $s+\Delta s$ from the initial point of the ray is given by

$$
I(s)-I(s+\Delta s)=I(s)\left(1-e^{-2 \alpha \Delta s}\right) .
$$

Our model assumes that this power loss has occurred in the middle point of this segment (i.e., the point at distance $s+\frac{1}{2} \Delta s$ ). It is then assigned to the nearest grid point in a rectangular grid. Rays are terminated if their energy is less than a fraction (default 0.01) of the initial energy of a ray starting at one of the elements. In this way a threedimensional table $\hat{Q}_{i j k}$ is computed, such that $\hat{Q}_{i j k}$ is the total power loss (in $\mathrm{W} / \mathrm{cm}^{3}$ ) in the volume element around the grid point $\left(x_{i}, y_{j}, z_{k}\right)$.

In fact the power loss does not only give rise to heat production, also other effects like scattering play a role. That means that the actual heat production density $Q_{i j k}$ is given by $Q_{i j k}=b \hat{Q}_{i j k}$, where $b$ is the absorption fraction. The value of absorption fraction $b$ depends on the tissue type and may be as low a 0.3 (Goss et al. 1979). The ray tracer and the computation of the heat production density were implemented in MATLAB (MathWorks, Natick, MA). 
2.1.2. Computation the temperature evolution Once the heat production density is known, the temperature $T$ is found by solving the heat equation

$$
\frac{\partial T(\mathbf{r}, t)}{\partial t}=D \Delta T(\mathbf{r}, t)+\frac{1}{\rho c_{p}} Q(\mathbf{r}, t)
$$

where $Q(\mathbf{r}, t)$ is the heat production density at position $\mathbf{r}$ and time $t$, and $D$ is the heat diffusion coefficient, given by $D=\frac{k_{\mathrm{T}}}{\rho c_{p}}$, with $k_{\mathrm{T}}$ the thermal conductivity, $\rho$ the density and $c_{p}$ the specific heat (at constant pressure), all depending on the material type. The value of $Q(\mathbf{r}, t)$ is obtained from the heat production table $Q_{i j k}$ (see subsection 2.1.1) and the sonication time. The sonication time is modeled by a step function, i.e., the heat source is described by $Q_{i j k}$ under sonication and 0 otherwise. For an in vivo situation, the heat transport by the perfusion has to be added to the heat source. We use von Neumann boundary conditions, which means that there is no heat flux through the boundaries of the system. The diffusion length in a time $\tau$ is approximately given by $\sqrt{4 D \tau}$. For a simulation with a total duration of $\tau=100 \mathrm{~s}$ and a diffusion coefficient in muscle tissue of $D=0.15 \mathrm{~mm}^{2} / \mathrm{s}$ that gives a length of only $7.7 \mathrm{~mm}$. Since the considered system is much larger than $7.7 \mathrm{~mm}$, the temperature at the system boundaries will hardly change in $100 \mathrm{~s}$, which justifies our assumption of no heat flux through the boundaries.

This heat equation can be solved by means of any standard finite-element software. We implemented our calculation in COMSOL Multiphysics (COMSOL, Inc., Burlington, $\mathrm{MA})$.

2.1.3. Larger sonication regions The previously described approach, with a $1 \mathrm{~mm}$ radius target region for the rays, is used to model a $2 \mathrm{~mm}$ diameter sonication cell. In practice larger regions are treated by superimposing $2 \mathrm{~mm}$ focal regions. A $4 \mathrm{~mm}$ diameter cell sonication is performed by superimposing $2 \mathrm{~mm}$ focal regions to cover the complete trajectory as shown in Figure 3. Since the (electronic) switching between the various $2 \mathrm{~mm}$ regions is very fast compared to the time scale of the temperature evolution, this process can be modeled by using one heat production density table $Q$, that is obtained by adding the tables for the individual $2 \mathrm{~mm}$ sub regions. This approach is not suitable for larger treatment cells. For instance, the $8 \mathrm{~mm}$ cell sonication in Figure 3 is performed by dividing it into an inner and an outer circle of basic treatment cells $(2 \mathrm{~mm})$ according to te scheme presented in Figure 3. This leads to two heat production densities: a table $Q_{i j k}^{(1)}$ corresponding to the inner trajectory of $4 \mathrm{~mm}$ diameter and a table $Q_{i j k}^{(2)}$ that corresponds to the outer trajectory of $8 \mathrm{~mm}$ in diameter. When solving the bio-heat equation the actual heat source $Q$ is, depending on the time, either $Q^{(1)}$ or $Q^{(2)}$.

\subsection{Comparisons}

2.2.1. Theoretical comparison with Far Field Approximation An alternative method to compute the heat production in one homogeneous material type is as follows. The pressure field of one transducer element is approximated by the far field 


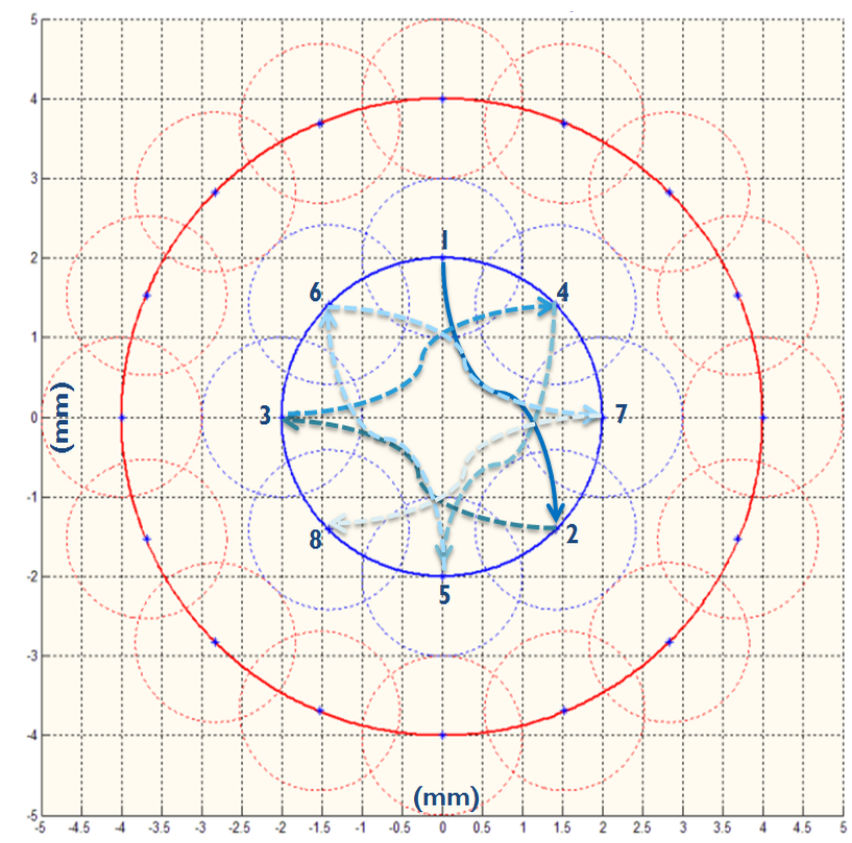

Figure 3. Position of the multiple-steered focal points in the intended ablated plane simulated in this study. Dashed circles are $2 \mathrm{~mm}$ diameter regions, solid curves are 8 $\mathrm{mm}$ (red) and $4 \mathrm{~mm}$ (blue) trajectories for multiple sonication. Arrow indicates the way switching order of the focal point around the inner trajectory as it is performed by the system

Table 1. Parameters used in the simulation comparison.

\begin{tabular}{|c|c|c|c|c|c|}
\hline $\begin{array}{c}f \\
{[\mathrm{MHz}]}\end{array}$ & $\begin{array}{c}a \\
{[\mathrm{~mm}]}\end{array}$ & $\begin{array}{c}S_{0} \\
{\left[\mathrm{~kg} /\left(\mathrm{m}^{2} \mathrm{~s}^{2}\right)\right]}\end{array}$ & $\begin{array}{c}\rho \\
{\left[\mathrm{kg} / \mathrm{m}^{3}\right]}\end{array}$ & $\begin{array}{c}c \\
{[\mathrm{~m} / \mathrm{s}]}\end{array}$ & $\begin{array}{c}\alpha \\
{[\mathrm{Np} / \mathrm{m}]}\end{array}$ \\
\hline 1.4 & 3.3 & $1.5007 \cdot 10^{7}$ & 1040 & 1537 & 5.76 \\
\hline
\end{tabular}

approximation (Morse 1986), which is a generally accepted approach. Then, by adding the pressure fields of all transducer elements, the total pressure field and the corresponding heat production is obtained. Consider a circular transducer element with radius $a$, centered in the origin and directed towards the positive $x$ axis, that emits acoustic waves with wave number $k=2 \pi f / c$, where $f$ is the frequency and $c$ the speed of sound. The far field approximation for the pressure field of this element at a position $\mathbf{r}=(x, y, z)$ is then given by

$$
p_{\text {ffa }}(\mathbf{r})=\pi a^{2} S_{0} \frac{e^{-i k r-\alpha r}}{2 \pi r} \frac{2 J_{1}(k a \sin (\theta)}{k a \sin (\theta)},
$$

where $r=|\mathbf{r}|, \theta$ is the angle between the vector $\mathbf{r}$ and the normal on the transducer element, $J_{1}$ is a Bessel function of the first kind, $\alpha$ is the (pressure) attenuation coefficient and $S_{0}$ is a measure for the strength of the element. The value of $S_{0}$ has been chosen such that the emitted power of each transducer element equals 1 watt, see section 2 of the suppl.info. 
Since the far field approximation assumes one homogeneous material type, this comparison is restricted to the case of a (theoretical) configuration with only one material type. For a theoretical comparison it is of no use to differentiate between absorption and attenuation. Hence we assume here that for both the ray tracer and the far field approximation the absorption fraction $b$ equals 1 . For that case the heat flux and the heat production, as computed by the ray tracing approach, are compared with the results from the far field approximation method. For the ray tracer the total heat flux through a coronal plane at distance $x$ is given by

$$
F_{\mathrm{R}}(x)=P_{\text {init }}-\int_{x_{\min }}^{x}\left(\iint \hat{Q}\left(x^{\prime}, y, z\right) d y d z\right) d x^{\prime}
$$

where $\hat{Q}(x, y, z)$ is the heat production density computed by the ray tracer at position $(x, y, z), P_{\text {init }}$ is the initial acoustic power and the material with attenuation starts at position $x_{\text {min }}$. The integral is computed by summation over the corresponding part of the heat production table $\hat{Q}_{i j k}$, see subsection 2.1.1. Grid sizes of $2 \mathrm{~mm}$ and $0.5 \mathrm{~mm}$ were used for the ray tracer and the far field approximation, respectively.

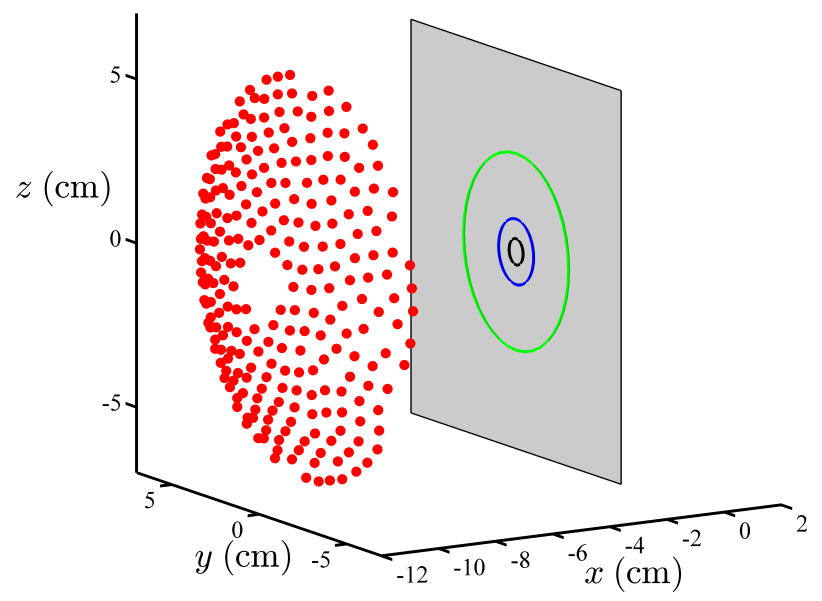

Figure 4. Heat production integrated over circles with radius $R$ in coronal planes at position $x$. Green circle: $R=3 \mathrm{~cm}$, blue circle $R=1 \mathrm{~cm}$, black circle $R=0.42 \mathrm{~cm}$.

Even if the total heat fluxes through coronal planes of the two methods agree well, the distribution of the heat flux (and hence heat production) in the coronal planes may not be similar. Therefore we also compared the integrated heat production over circles of with radius $R$ (in $\mathrm{W} / \mathrm{cm}$ ) in coronal planes, see also Figure 4 . We used circles with radius $R=8 \mathrm{~cm}, 3 \mathrm{~cm}, 1 \mathrm{~cm}$ or $0.4 \mathrm{~cm}$. For both the ray tracer and the far field approximation method the integrated heat production over parts of coronal planes can be computed by integrating (summing up) over the corresponding part of heat production densities $Q$ and $Q_{\mathrm{F}}$ respectively.

For both methods the heat production was computed for $-10 \mathrm{~cm} \leq x \leq 2 \mathrm{~cm}$ and $-8 \mathrm{~cm} \leq y, z \leq 8 \mathrm{~cm}$, where the transducer is in the negative $x$ direction, the focal distance is $12 \mathrm{~cm}$ and the natural focus point is in the origin, see also Figure 2 for the 
used coordinates. The used parameter values are given in Table 1. More details of the flux computation based on the far field approximation are given in Section 1 of the suppl. info.

2.2.2. Contribution of shear waves. To assess contribution of shear waves to the total heat production in bone, two types of numerical simulations were performed. In the first simulations we compared the standard heat production in a bone to the hypothetical situation where the bone behaves acoustically as a liquid. In that case shear waves in bone do not exist and a much larger fraction of the acoustic energy is reflected.

For a bone a mesh was generated (by COMSOL) with a minimum element size of $0.1 \mathrm{~cm}$ and a maximum element size of $1 \mathrm{~cm}$. The smaller elements were used in the focal region, where most of the heat is produced. In the mesh, two nested coaxial cylinders with radiuses of $1.0 \mathrm{~cm}$ and $2.0 \mathrm{~cm}$, respectively, and height of $15 \mathrm{~cm}$ represents the marrow and the bone. (Thus, the thickness of the bone containing the marrow is $1 \mathrm{~cm}$.) The bone cylinder is encased in a box with muscle properties with dimensions $\mathrm{L} \times \mathrm{W} \times \mathrm{H}$ of $15 \times 15 \times 15 \mathrm{~cm}^{3}$. Finally this box is nested within another box with water properties and dimensions $20 \times 20 \times 15 \mathrm{~cm}^{3}$. The distance from the transducer to the focus point and the center of the mesh are $15 \mathrm{~cm}$ and $16.5 \mathrm{~cm}$, respectively. The whole object can be rotated around the z-axis. The tilt of the object is measured by the angle $\gamma$ with the $z$-axis. The configuration is similar to the one in Figure 2. To be able to clearly show the tilt, the encasing muscle and water meshes are not shown in Figure 2,

The rotation angle $\gamma$, i.e., the inclination of the mesh with respect to the z-axis, was varied in the range $-55^{\circ}$ to $55^{\circ}$ divided in steps of one degree. For each angle $\gamma$ the transducer power was $30 \mathrm{~W}$. The parameters for this experiment are given in Table 2. To avoid computing the almost vanishing heat production in water and oil (due to the very small attenuation coefficients $\alpha$ ) we have set the absorption fraction $b$ to 0 for both materials. The heat production was calculated using a grid with a spacing of $0.1 \mathrm{~cm}$ only in the points residing in the bone cylinder. For each angle the ratio was computed between the total heat productions in the bone without and with shear waves. In the former case, to eliminate the shear waves, the bone was treated as liquid.

In the second numerical experiment we investigated the effect of omitting shear waves on the resulting temperatures. Bone was defined as a cylinder of $10 \mathrm{~cm}$ height, $0.5 \mathrm{~cm}$ external radius and $0.2 \mathrm{~cm}$ internal radius embedded in a box of $2 \mathrm{x} 2 \mathrm{~cm}^{2}$ sides and $10 \mathrm{~cm}$ height. The surrounding box was defined as tissue mimicking gel. The distance transducer to bone and the focal distance were set to $13.5 \mathrm{~cm}$. Bone geometry was meshed with a minimum element size of $0.1 \mathrm{~cm}$. The computation was run for a fixed power of $120 \mathrm{~W}$ and two angles: a normal incidence and $50^{\circ}$ incidence (close to total reflection limit). The other parameters where as in Table 2, except the speed of sound in the tissue mimicking gel, which was $1537 \mathrm{~m} / \mathrm{s}$ in this simulation. The heat production was computed for each angle including and omitting shear waves mode-conversion. The heat equation solution was solved for each case considering 60 s sonication time followed 
Table 2. Parameters used in simulations on shear waves and in the gel-bone comparison

\begin{tabular}{|c|c|c|c|c|c|c|}
\hline material & $\begin{array}{c}c \\
{[\mathrm{~m} / \mathrm{s}]}\end{array}$ & $\begin{array}{c}\rho \\
{\left[\mathrm{kg} / \mathrm{m}^{3}\right]}\end{array}$ & $\begin{array}{c}c_{p} \\
{[\mathrm{~J} /(\operatorname{kg~K})]}\end{array}$ & $\begin{array}{c}k_{\mathrm{T}} \\
{[\mathrm{W} /(\mathrm{m} \mathrm{K})]}\end{array}$ & $\begin{array}{c}\alpha \\
{[\mathrm{Np} / \mathrm{cm}]}\end{array}$ & $b$ \\
\hline gel & 1492 & 1030 & 3720 & 0.537 & 0.07 & $0.35^{1}$ \\
\hline bone (longit) ${ }^{2}$ & 3736 & 2025 & 1300 & 0.487 & 1.9 & $0.3^{3}$ \\
\hline bone $(\text { shear })^{2}$ & 1995 & 2025 & 1300 & 0.487 & 2.8 & $0.3^{3}$ \\
\hline water & 1523 & 997 & - & - & $6.08 \cdot 10^{-4}$ & 0 \\
\hline oil & 1380 & 1030 & - & - & 0 & 0 \\
\hline \multicolumn{7}{|c|}{$\begin{array}{l}\text { - the specific heat and thermal conductivity for water and oil are not needed, } \\
\text { as solving the heat equation in these materials is unnecessary. } \\
{ }^{1} \text { average of different values in Goss et al. (1979) } \\
{ }^{2} \text { Nell \& Meyers (2010) } \\
{ }^{3} \text { Pinton et al. (2012) }\end{array}$} \\
\hline
\end{tabular}

by $60 \mathrm{~s}$ cooling time.
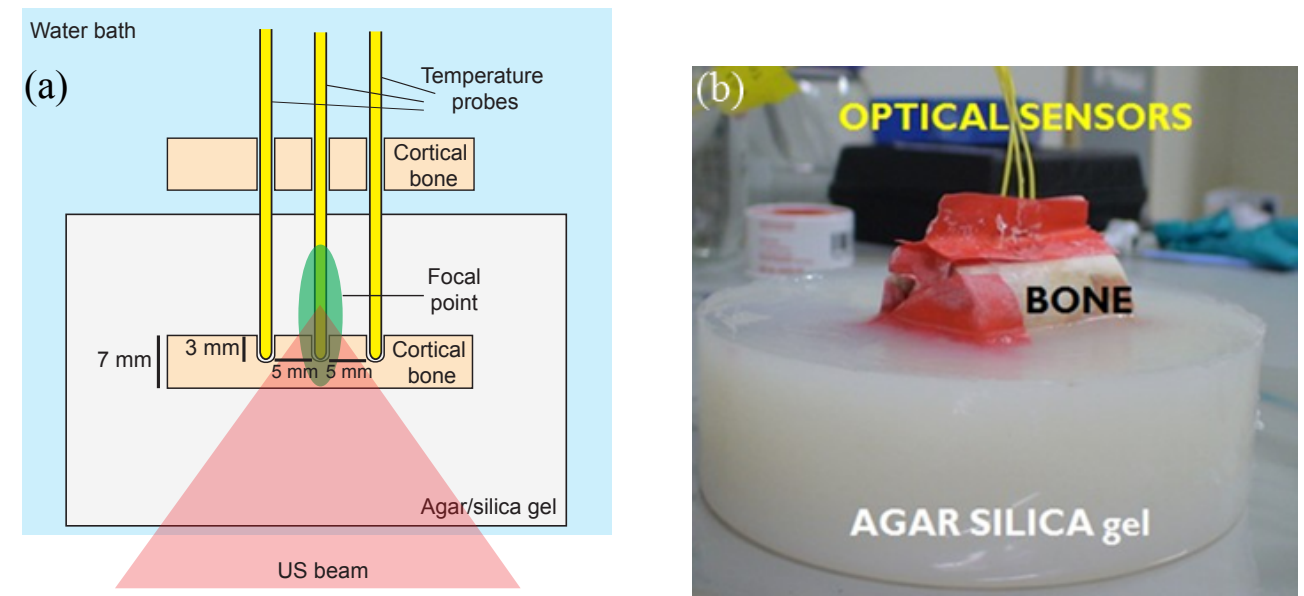

Figure 5. Set up for a bone phantom ablation experiment.(a): Schematic (b): Photograph

2.2.3. Comparison with bone phantom experiments As a proof of concept, a bone phantom was designed matching a simple geometrical model. A pig femur was sized into a cylindrical shape (Figure 5 a) with a $0.7 \mathrm{~cm}$ cortex thickness. For temperature measurements inside the bone, 3 holes were drilled from the inside up to $3 \mathrm{~mm}$ below the cortex surface, to position MR compatible optical temperature sensors (Neoptix T1 probes, Quebec City, Canada). The accuracy of the sensors is tested under different conditions (warm and hot water, free and within bone), using a laboratory thermometer as a reference.

The bone was positioned in a beaker and then tissue mimicking agar-silica gel was added, to obtain the final setup as shown in Figure 5b. The gel was prepared using $20 \mathrm{~g}$ agar and $30 \mathrm{~g}$ silica per liter water, resulting in density, speed of sound and attenuation 
as given in Table 1 (Partanen et al. 2009). The phantom was positioned on a clinical HIFU table (Philips Sonalleve, Vantaa, Finland) in direct contact with the membrane on the tabletop, with water and additional gel pads to ensure acoustic coupling, in the center of a $3 \mathrm{~T}$ MRI scanner (Philips Achieva, Best, the Netherlands).

An MR-HIFU ablation experiment was performed using a protocol that is comparable to currently clinically used protocols for pain palliation procedures. For this protocol a $8 \mathrm{~mm}$ treatment cell (see subsection 2.1.3) was positioned $1 \mathrm{~cm}$ behind the bone surface. The HIFU transducer produced 110 watt during 27 seconds, i.e. approximately $3 \mathrm{~kJ}$ energy. The incident angle was defined to be zero relative to the normal of the bone surface, as shown in Figure 6. The focal region (Figure 4a) was positioned along the central temperature sensor direction, thus measuring the temperature increase in the hottest region. The other sensors were positioned only 0.5 $\mathrm{cm}$ apart from each other to ensure that the heat diffusion could be monitored in time. Data from the sensors were recorded by a LabVIEW program (LabVIEW, National Instruments). MR-thermometry was performed using the PRFS. technique (Rieke \& Butts Pauly 2008, Köhler et al. 2009, McDannold 2005, Denis De Senneville et al. 2005) which is the state of the art for MR thermometry. Temperature maps were measured in 6 slices (see Fig. 4b), and every 6 seconds a full temperature map for these slices were acquired (for more details see table 3). MR-thermometry and data from temperature sensors were afterwards compared with simulations. Parameters used in the simulations are listed in Table 2. To demonstrate the robustness of this model, three comparable experiments were performed using a $4 \mathrm{~mm}$ treatment cell, an acoustic power of $60 \mathrm{~W}$, a treatment duration of $20 \mathrm{~s}$ and a frequency of $1.2 \mathrm{MHz}$. The exact experimental setup is described in the supplementary information under Section 4.

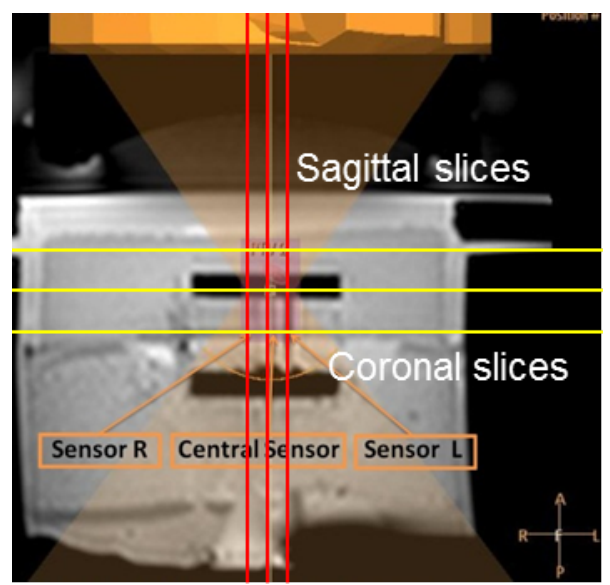

Figure 6. Planning of bone ablation procedure using T2-weighted MR-images, where white is the Agar-Silica Gel and black is Bone (parallel to coronal slices). Red and Yellow are the slices where temperature monitoring is performed. The HIFU focus is at the cross section of the central sagittal and coronal slice. 
Table 3. MR Thermometry - sequence parameters

\begin{tabular}{|l|l|}
\hline MR Sequence M2D-EPI & $\mathrm{TR} / \mathrm{TE}=38 / 20 \mathrm{~ms}$ \\
\hline Spatial Resolution & $1.42 \times 1.42 \times 4.08 \mathrm{~mm}^{3}$ \\
\hline Temporal resolution & 6 slices in 6.1 seconds \\
\hline
\end{tabular}

\section{Results}

\subsection{Theoretical comparison with Far Field Approximation}

In Figure 7 the total power flux through coronal planes at position $x$ as computed with the far field approximation $\left(F_{\mathrm{F}}(x)\right)$ and with the ray tracer $\left(F_{\mathrm{R}}(x)\right)$ are shown. Also a line with the decay $C e^{-2 \alpha x}$ (with $C$ a constant) is shown. This exponential decay is found if the distance that a ray travel through the gel is approximated by the horizontal distance $(x)$.

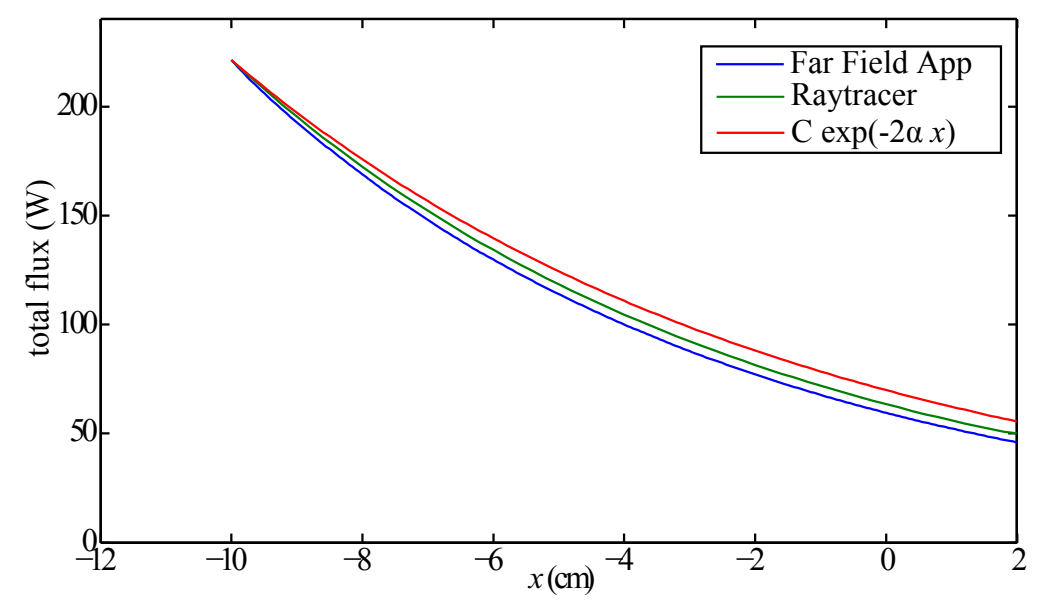

Figure 7. The heat flux computed by the far field approximation (blue) and by the ray tracer (green), and a curve with exponential decay according to $C e^{-2 \alpha x}$ (red). Total power $256 \mathrm{~W}$, focal distance $12 \mathrm{~cm}$.

The comparison of the integrated heat production over circles in coronal planes is shown in Figure 8, as function of the position $x$ of the coronal plane. The two red lines in Figure 8 give the total heat production within $8 \mathrm{~cm}$ of the center. All transducer elements are within a distance of $8 \mathrm{~cm}$ from the $x$-axis and focus towards the $x$-axis, hence the red lines give the total heat produced in the whole coronal plane, computed by both methods. Since the heat flux computed by both methods is almost identical (see Figure 7), this also holds for the heat production within circles of radius $R=8 \mathrm{~cm}$. However, for decreasing radii $R$ the ray tracer predicts an increasingly higher heat production compared to the far field method. The computation time for the ray tracer approach was in the order of minutes while the far field approximation method took about 5 days, using the same computer (Matlab under Windows 7 on Intel Core i7-3770 $3.4 \mathrm{GHz}$, with 8GB RAM). 


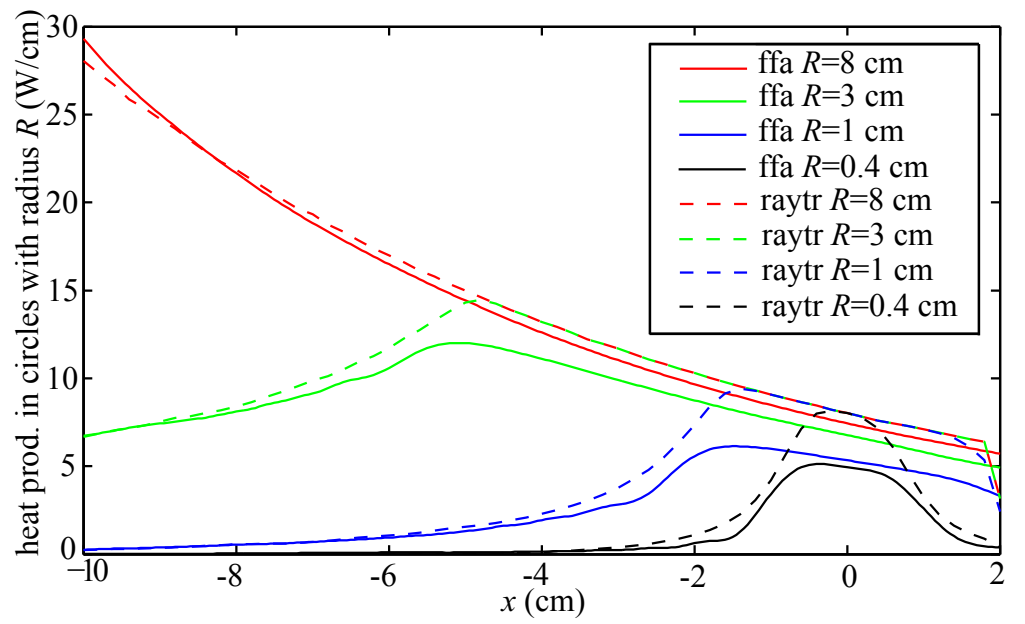

Figure 8. Integrated heat production in circles with radius $R$ in coronal planes. Red lines: $R=8 \mathrm{~cm}$, green lines: $R=3 \mathrm{~cm}$, blue lines $R=1 \mathrm{~cm}$, black lines $R=0.4 \mathrm{~cm}$. Total power 256 watt, focal distance $12 \mathrm{~cm}$.

\subsection{Contribution of shear waves}

The importance of shear waves in bone can already be seen from the reflection/transmission coefficients at the muscle-bone interface, as shown in Figure 1. For comparison, Figure 9 shows the reflection/transmission coefficients computed for the muscle-bone interface without taking shear waves in the bone into account, i.e., considering it as a liquid-liquid interface. For incoming angles $\theta_{i l}$ up to about $24^{\circ}$ both the actual liquid-solid interface and the hypothetical liquid-liquid interface show transmission of energy into the bone. For incoming angles between about $24^{\circ}$ and $52^{\circ}$ the highest difference between the two approaches is observed. Excluding shear waves (Figure 9) leads to no energy transmission into the bone above the angle of total reflection (ca $24^{\circ}$ ), while in fact more than $75 \%$ of the energy enters the bone as shear wave (Figure 1).

Figure 10 shows the results of the simulations concerning the contribution of shear waves on tilted bone, taking the actual transducer geometry into account. The results show that for a sonication with the transducer perpendicular to the bone $(\gamma=0)$, omitting shear waves leads to a heat production in the bone that is about $30 \%$ of the heat production including shear waves. This can be explained by noting that even for a transducer perpendicular to the bone, the rays from many transducer elements are not perpendicular to the bone. Moreover, the bone surface is curved. For tilted bone the effect of omitting shear waves becomes more pronounced. For angles $|\gamma| \geq 46^{\circ}$ there is even no heat transferred to the bone by longitudinal waves.

Figure 11 shows the temperatures calculated in the second numerical experiment for the three selected points in soft tissue in muscle in front of the bone $(0.5 \mathrm{~cm})$, inside the bone cortex and in the bone marrow during a sonication with (a) and without (b) inclusion of shear waves. The temperature in the cortical bone after $60 \mathrm{sec}$ sonication and $30 \mathrm{~W}$ power is about $110^{\circ} \mathrm{C}$ without shear waves and more than $160^{\circ} \mathrm{C}$ with shear 


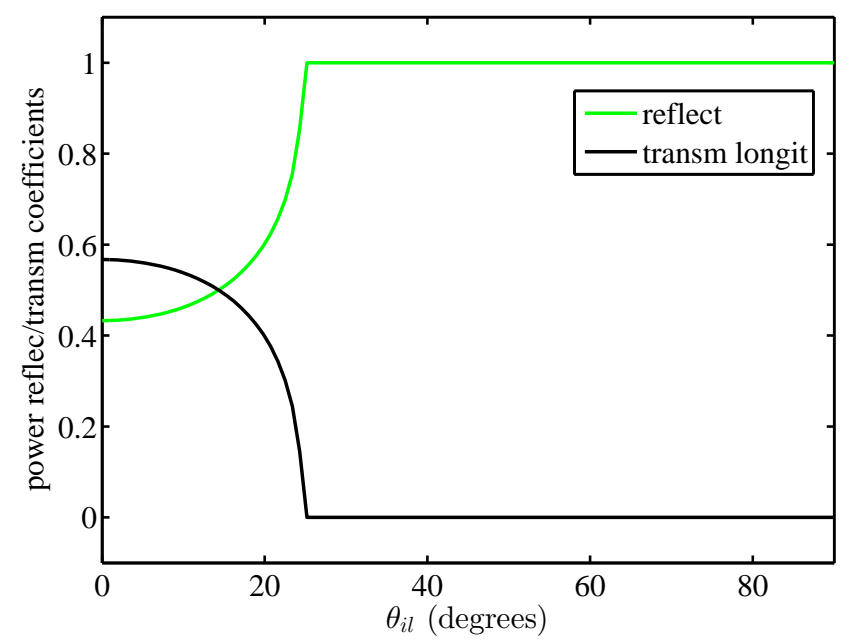

Figure 9. Reflection/transmission coefficients at a hypothetical muscle-bone interface without shear waves in bone.

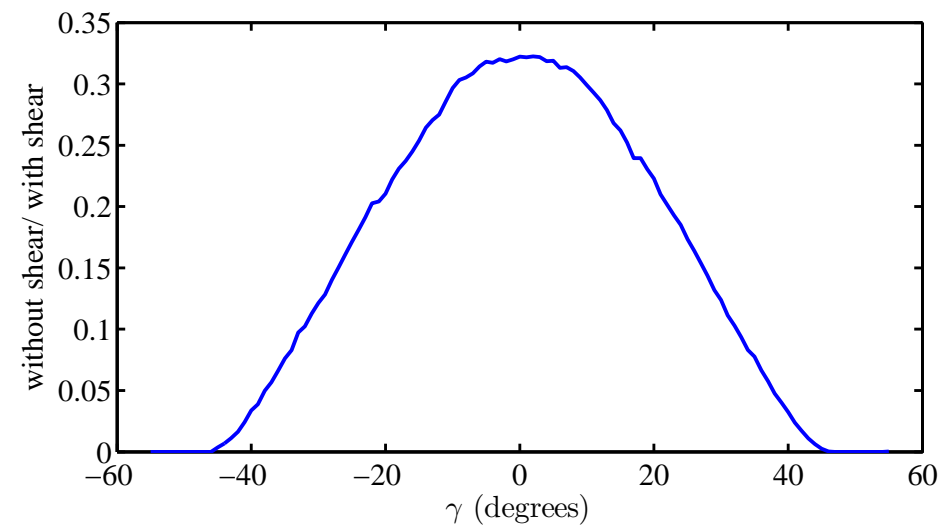

Figure 10. Heat production ratio in bone for the cases without and with shear waves as function of the bone inclination angle $\gamma$.

waves. A computation shows that, even with normal incidence, the rays from several outermost transducer elements have an angle of more than $32^{\circ}$ with the normal on the bone. Hence in the situation without shear waves these rays are totally reflected.

For the case of $50^{\circ}$ incidence (Figure 12 ) the computation without shear waves predicts for the cortical bone a maximum temperature of about $43^{\circ} \mathrm{C}$ while the computation with shear waves predicts a temperature of more than $100^{\circ} \mathrm{C}$. As in the case with shear waves only rays with an angle of more than $52^{\circ}$ lead to total reflection, most rays at an angle of $50^{\circ}$ incidence still contribute via generating shear waves to the heating of the bone. Clearly omitting shear waves leads to low accuracy in the prediction of temperature in the bone and a smaller spread at different locations in the sonication area. 

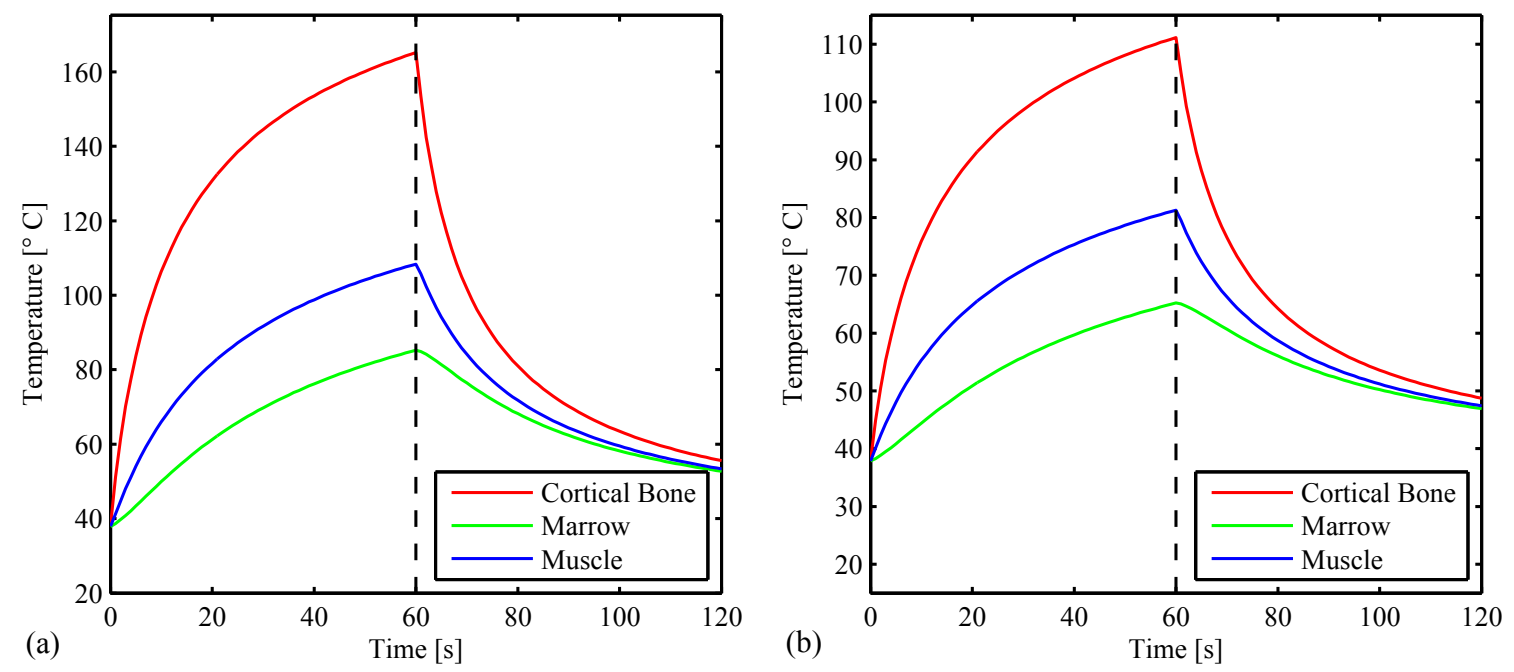

Figure 11. Comparison of reached temperatures with shear waves (a) and without shear waves (b) in bone, for normal incidence.
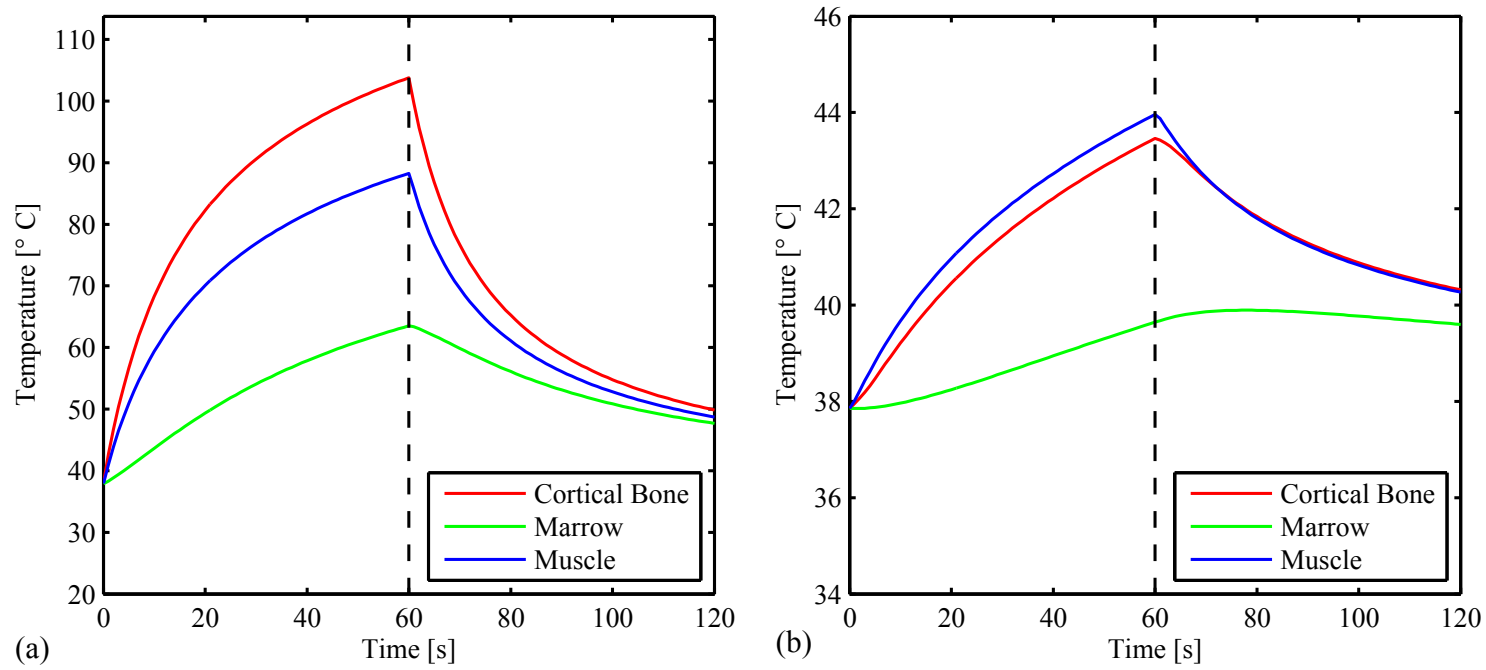

Figure 12. Comparison of reached temperatures with shear waves (a) and without shear waves (b) in bone, for incidence with $50^{\circ}$.

\subsection{Comparison with bone phantom experiments}

For a first comparison of the modeling approach with experimental data, one HIFU ablation was performed using a bone embedded in a tissue mimicking gel. The setup of bone-phantom and the sensor-positions were shown in Figure 6 with sensors positioned $5 \mathrm{~mm}$ apart and are inserted in the cortex $3 \mathrm{~mm}$ behind the tissue bone surface. A sonication with $110 \mathrm{~W}$ for 27 seconds was performed on the bone phantom with normal incidence using an $8 \mathrm{~mm}$ treatment cell, with the focus placed $1 \mathrm{~cm}$ behind the cortex into the bone marrow. Figure 13 shows the experimental temperature maps in the tissue mimicking gel that were acquired using MR PRFS temperature mapping, in comparison 
to computed temperature maps, 30 seconds after start of the sonication. Figure 14 shows the temperature profiles along the line in the central plane and in addition the result of the central temperature sensor placed in the cortex. Finally, Fig. 15 compares the temperatures measured with sensors to the simulation results as function of the time. The breakpoint in simulation $\mathrm{C}$ at $t=18 \mathrm{sec}$ is a consequence of the $8 \mathrm{~mm}$ treatment cell, see subsection 2.1 .3 .

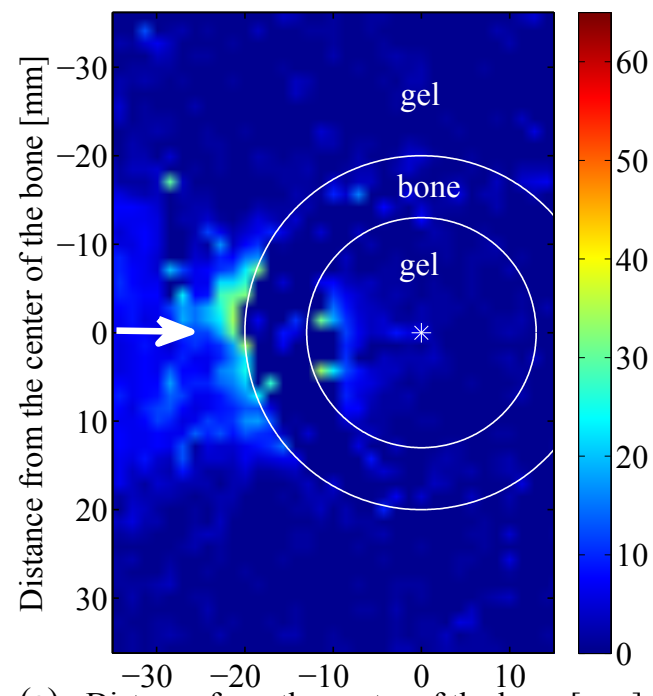

(a) Distance from the center of the bone $[\mathrm{mm}]$

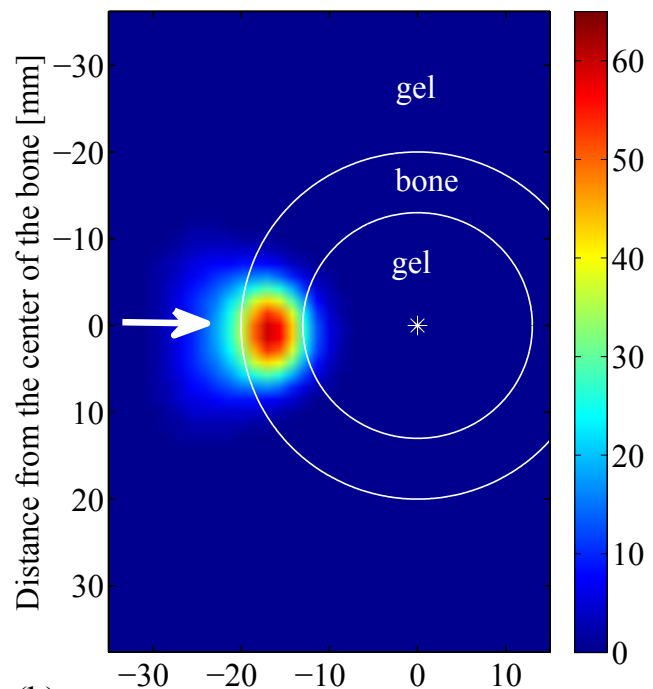

(b) Distance from the center of the bone $[\mathrm{mm}]$

Figure 13. Bone-phantom experiment. Experimental (a) and computed (b) temperatures in sagittal plane at $30 \mathrm{~s}$ after start of sonication. Note the absence of experimental temperature data in the bone.

Note that, since MRI thermometry cannot measure the temperature in bone, the MR-based experimental temperature data in bone are not relevant.

We also investigated the sensitivity of the temperatures with respect to the position of the focal point and the sensor. In our experiments slice thickness was 1.42 and 4.08 $\mathrm{mm}$ in the lateral and axial direction respectively (see Table 3). Simulations showed that mis-positioning of MR thermometry slices of a 2-4 mm leads up to $10 \%$ temperature difference in the lateral directions and up to $20 \%$ along the beam axis. The temperature sensor used for bone temperature measurement is affected by its relative position to the focal region. A lateral shift of $2 \mathrm{~mm}$ leads up to $15 \%$ difference in the temperature estimation in the bone. Additional results comparing modeling and experimental data can be found in the supplementary information under Section 4.

\section{Discussion}

Our aim was to develop an efficient and feasible method to compute the temperature evolution under HIFU. The computation of the heat production by HIFU, can in principle be performed by solving an adequate wave equation or Helmholtz equation, including a damping term. Corresponding boundary conditions and reflection, refraction 


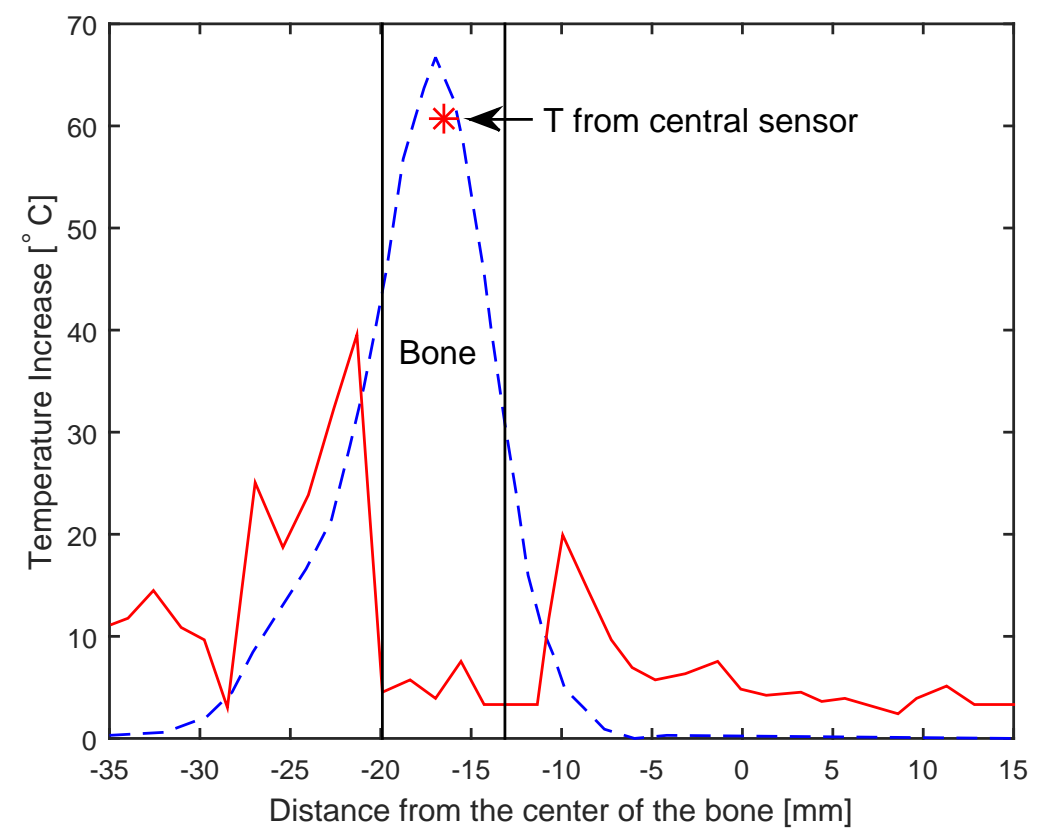

Figure 14. Temperatures along beam direction $30 \mathrm{~s}$ after start of sonication, obtained with PRFS-MR (solid line) measurement and sensor placed in the center of the cortical bone (star) in a bone phantom during MR-HIFU sonication in comparison to data obtained with ray tracing-based computation (dashed line).

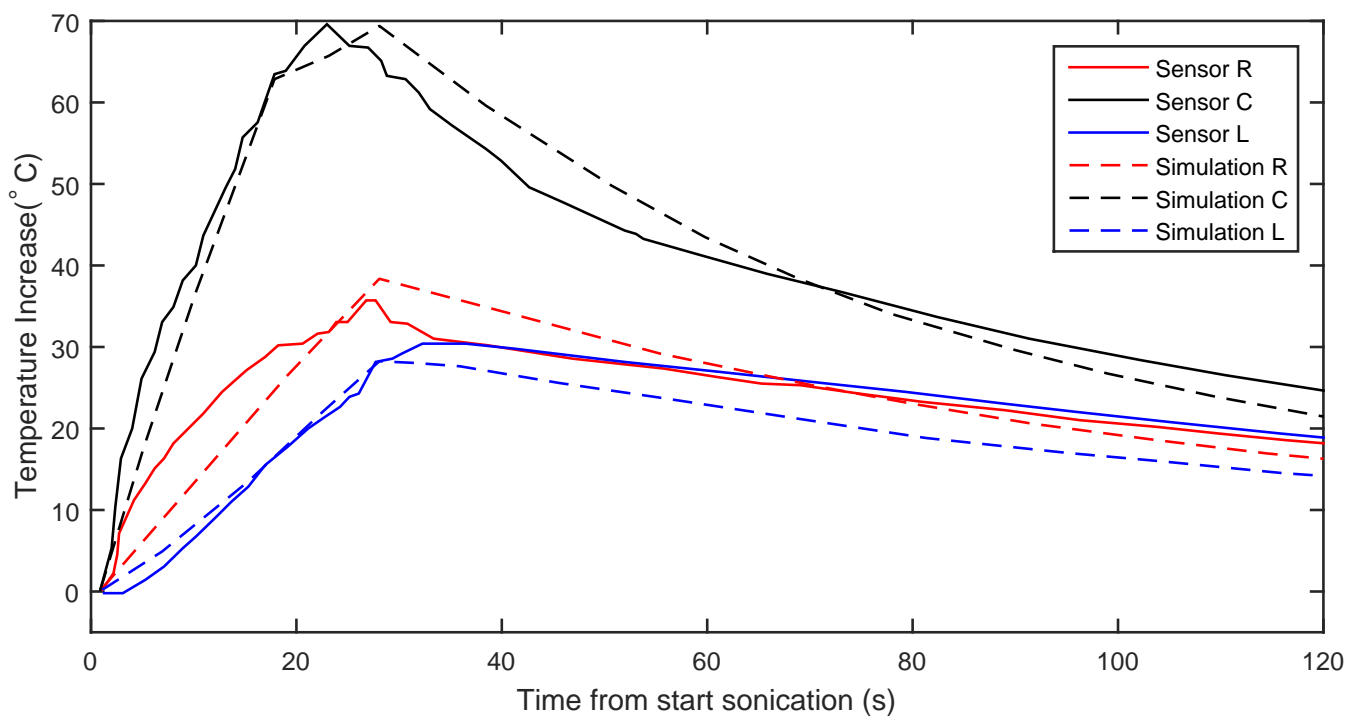

Figure 15. Temperature profiles. Comparisons between measurement data from sensors (solid line) and simulations (dashed line).

and mode conversion at interfaces between various materials must be included. Moreover, since the numerical grid size must also be considerably smaller than the 
wave length of the used ultrasound in muscle tissue, which is in our case about $1.3 \mathrm{~mm}$ $(f=1.4 \mathrm{MHz})$, solving a wave equation or Helmholtz equation requires a very large number of grid points and consequently, impractical computing times. This worsens in the presence of complex geometries and shear waves in solids. Therefore we opted for a ray tracing approach for a near-real time computation of heat production induced by HIFU. When applied to the bone-phantom configuration considered in Section 2.2.3 the running time of a $\mathrm{C}++$ implementation of the ray tracer is about 2 seconds range for more than 3 millions of rays. (Windows 8 on Intel 3550k i5, 16 GB memory, NVIDIA GeForce 660 GTX graphics processor (GPU)). Solving the resulting bio-heat equation with a finite difference method on a $128^{3}$ nodes grid on the same PC was done in about $76 \mathrm{sec}$, which is halved when the GPU is used. Our ray tracer accepts both simple geometrical shapes (cylinders and cubes) and more complex (bone) geometries, described by a triangular mesh. The latter allows the use of real patient scan data into this approach.

Figure 7 shows that the acoustic power fluxes computed with the far field approximation and with the ray tracer show only a minor difference. Hence the total flux through coronal planes is predicted well by the ray tracer. The exponential decay $C e^{-2 \alpha x}$ underestimates the decay, since the actual length of rays to a coronal plane at coordinate $x$ is more than $x$. Hence the actual decay is larger than this exponential decay, as indeed can be seen in Figure 7. However, the ray tracer overestimates the heat production in the focus region, see Fig. 8. This effect may be explained by the focussing of the rays on randomly selected points within a $1 \mathrm{~mm}$ radius circle in the focal plane and by the fact that the rays, as in the geometric optics equivalent, do not carry a phase, so interference is not considered. However, when applied to bone, with the focus point in the bone, the overestimation is much smaller. A reasonable explanation for this is that the attenuation in bone is much higher than in muscle, resulting in a strong decrease of the wave amplitudes before the focal region is reached.

The inclusion of shear waves and mode-conversion is of crucial importance for bone HIFU interaction. Figure 1 shows that for incoming angles between approximately $24^{\circ}$ and $52^{\circ}$ only shear waves, with about $75 \%$ of the energy. In fact the energy fraction transferred into the bone in the $24^{\circ}$ to $52^{\circ}$ range by shear waves is larger than the total energy fraction transferred into the bone for angles below $24^{\circ}$. Hence, in particular for oblique incidence shear waves are important. Note that even a transducer beam with perpendicular incidence on a cylindrical bone will result in rays with a variety of incoming angles and therefore to shear waves contributing to the heat production in bone. Furthermore, a non - flat transducer geometry will also contribute to incoming angle widening. For the transducer used in our experiments (Philips Sonalleve) the outermost transducer elements have already an incoming angle with (the normal to) a coronal plane in the focal region of more than $32^{\circ}$. The importance of shear waves is confirmed in the numerical experiments with tilted bone. Omitting shear waves reduces for normal incidence the total heat production in the bone to about 35\% (see Figure 10). This percentage falls to 0 for incidence angles around $47^{\circ}$. The same effect is shown 
in the numerical experiments that compute the temperature profiles for $0^{\circ}$ and $50^{\circ}$ incidence. For $0^{\circ}$ incidence Figure 11 shows that omitting shear waves leads to much lower temperatures. For the $50^{\circ}$ incidence case the effect is even larger (Figure 12).

In a proof of concept study, a first comparison of the simulation data with experimental data obtained during an HIFU ablation of bone embedded in a tissue mimicking phantom was performed. Figures 13,14 and 15 show that model data are in fair agreement with the experimental data, in particular when considering the experimental challenges, such as partial volume effects, dimensions of the voxels used for PRFS thermometry relative to the temperature gradient near bone, the inherent sensitivity of the PRFS technique to magnetic field stability and the influence of the accuracy of positioning the focal point on the temperature distribution. Also, additional bone phantom experiments described under Section 4 of the supplementary information demonstrated that the temperatures estimated by our model were in good agreement with those measured by the temperature sensors. An extensive comparison of the simulation results with the experimental data is ongoing. All above explains the considerable noise in the experimental data shown in Figures 13 and 14 for PRFSbased thermometry data. Moreover, simulations showed that the inaccuracy of both the focal point and the sensor position may lead to temperature deviations, as mentioned in Section 3.3. Our model could be further improved by taking non-linear effects of ultrasound in bonce into account, which could lead to additional heating of the bone cortex (Pinton et al. 2011). The importance and extend of non-linearity effects is currently under investigation.

Several earlier contributions model the interaction of HIFU with the tissue-bone interface (Lu et al. 2000, Myers 2004, Hynynen 1988, Hipp et al. 2012, Scott et al. 2013a). Fujii et al. (1999), Lin et al. (2000), and Nell \& Meyers (2010) deal also with the effect of shear waves. A common feature in these papers is the flat tissue-bone interface, which means that they cannot cope with realistic bone geometries. Fujii et al. (1999) already notes the importance of shear waves in case of oblique incidence. Lin et al. (2000) derive an analytical steady state solution of the bio-heat equation for a simplified onedimensional case, also for oblique incidence. In Nell \& Meyers (2010) a finite elements approach for the ultrasound field has been used and temperature evolution in bone is computed and compared with experiments. Compared to our findings they reported a significantly smaller contribution of shear waves, which is probably due to their smaller transducer, normal beam incidence and flat tissue-bone interface. Scott et al. (2013b) and Scott et al. (2014) study various modelling approaches for interstitial ultrasound ablation, including models that take shear waves into account.

Our ray tracer was applied to simple geometrical shapes (i.e. cylinders) but can also be used with more complex geometries, described by meshes. This approach allows to apply our model also to real bone lesions of patients having complicated morphologies, as the exact structure could be measured with computed tomography and rendered into a mesh. As the ray tracing approach allows a very fast computation, it may be extended to a application that can be used for patient selection and treatment planning. 


\section{Acknowledgements}

This work was partly performed within the framework of CTMM, the Center for Translational Molecular Medicine (www. ctmm.nl), project HIFU-CHEM (grant 03O301).

\section{References}

Aubry, J.-F., Pauly, K., Moonen, C., ter Haar, G., Mario Ries, M., Salomir, R., Sokka, S., Sekins, K., Shapira, Y., Ye, F., Huff-Simonin, H., Eames, M., Hanane, A., Kassel, N., Napoli, A., Hwang, J., Wu, F., Zhang, L., Melzer, A., Kim, Y., \& Gedroyc, W. (2013). The road to clinical use of high-intensity focused ultrasound for liver cancer: technical and clinical consensus, Journal of Therapeutic Ultrasound $\mathbf{1}(13)$.

Chen, W., Zhu, H., Zhang, L., Li, K., Su, H., Jin, C., Zhou, K., Bai, J., Wu, F. \& Wang, Z. (2010). Primary bone malignancy: effective treatment with high-intensity focused ultrasound ablation, Radiology 255(3): 967-978.

Coleman, R. E. (2006). Clinical features of metastatic bone disease and risk of skeletal morbidity, Clinical Cancer Research 12(20): 6243s-6249s.

Davis, R. M. \& Warren, W. S. (2014). Intermolecular zero quantum coherences enable accurate temperature imaging in red bone marrow, Magn Reson Med . doi: 10.1002/mrm.25372.

Denis De Senneville, B., B., Q. \& Moonen, C. (2005). Magnetic resonance temperature imaging, Int. J. Hyperthermia 21(6): 515-531.

Filippiadis, D. K., Tutton, S. \& Kelekis, A. (2014). Percutaneous bone lesion ablation, Radiol Med 119(7): 462-9.

Foley, J., Eames, M., Snell, J., Hananel, A., Kassell, N. \& Aubry, J. (2013). Image-guided focused ultrasound: state of the technology and the challenges that lie ahead, Imaging in Medicine 5(4): $357-370$.

Fujii, M., Sakamoto, K., Toda, Y., Negishi, A. \& Kanai, H. (1999). Study of the Cause of the Temperature Rise at the Muscle-Bone Interface During Ultrasound Hyperthermia, IEEE Transactions on Biomedical Engineering 46(5): 494-404.

Goss, S., Frizzel, L. \& Dunn, F. (1979). Ultrasound Absorption and Attenuation in Mammalian tissues, Ultrasound in medicine 83 biology 5: 181-186.

Haar, G. \& Coussios, C. (2007). High intensity focused ultrasound: physical principles and devices, Int. J. Hyperthermia 23(2): 89-104.

Hipp, E., Partanen, A., Karczmar, G. \& Fan, X. (2012). Safety limitations of MR-HIFU treatment near interfaces: a phantom validation, Journal of Applied Clinical Medical Physics 13(2): 168-175.

Huisman, M., Lam, M., Bartels, L., Nijenhuis, R., Moonen, C., Knuttel, F., Verkooijen, H., van Vulpen, M. \& van den Bosch, M. (2014). Feasibility of volumetric mri-guided high intensity focused ultrasound (mr-hifu) for painful bone metastases, Journal of Therapeutic Ultrasound 2(1): 16.

Huisman, M. \& van den Bosch, M. A. A. J. (2011). Mr-guided high-intensity focused ultrasound for noninvasive cancer treatment, Cancer Imaging 11(1A): S161-S166.

Hurwitz, M. e. a. (2014). Magnetic Resonance: Guided Focused Ultrasound for Patients With Painful Bone Metastases: Phase III Trial Results, J Natl Cancer Inst 106(5).

Hynynen, K. (1988). Temperature elevation at muscle-bone interface during scanned, focused ultrasound hyperthermia, Int. J. Hyperthermia 4(3): 267-79.

Hynynen, K. (2010). MRI-guided focused ultrasound treatments, Ultrasonics 50(2): 221-229.

Köhler, M., Mougenot, C., Quesson, B., Enholm, J., Le Bail, B., Laurent, C., Moonen, C. \& Ehnholm, G. (2009). Volumetric HIFU ablation under 3D guidance of rapid MRI thermometry, Med. Physics 36: 3521-3535. 
Kurup, A. \& Callstrom, M. (2010). Ablation of skeletal metastases: Current status, J Vasc Interv Radiol 21(8): S242-S250.

Li, C., Zhang, W., Fan, W., Huang, J., Zhang, F. \& Wu, P. (2010). Noninvasive treatment of malignant bone tumors using high-intensity focused ultrasound, Cancer 116(16): 3934-3942.

Liberman, B., Gianfelice, D., Inbar, Y., Beck, A., Rabin, T., Shabshin, N., Chander, G., Hengst, S., Pfeffer, R., Chechick, A., Hanannel, A., Dogadkin, O. \& R., C. (2009). Pain palliation in patients with bone metastases using mr-guided focused ultrasound surgery: a multicenter study, Ann Surg Oncol 16(1): 140-6.

Lin, W., Liauh, C., Chen, Y., Liu, H. \& Shieh, M. (2000). "Theoretical study of temperature elevation at muscle/bone interface during ultrasound hyperthermia, Med. Physics 27(5): 1131-1140.

Lu, B., Yang, R., Lin, W., Cheng, K., Wang, C. \& Kuo, T. (2000). Theoretical study of convergent ultrasound hyperthermia for treating bone tumors, Med. Eng. Phys 22(4): 253-63.

McDannold, N. (2005). Quantitative mri-based temperature mapping based on the pro- ton resonant frequency shift: review of validation studies, Int. J. Hyperthermia 21(6): 533-546.

Moros, E. G., Straube, W., Myerson, R. \& Fan, X. (2000). The impact of ultrasonic parameters on chest wall hyperthermia, Int. J. Hyperthermia 16(6): 523-538.

Moros, E. G., Xiaobing, F. \& Straube, W. (1999). Ultrasound power deposition model for the chest wall, Ultrasound in medicine 83 biology 25(8): 1275-1287.

Morse, P. (1986). Theoretical Acoustics, Princeton University Press.

Myers, M. R. (2004). Transient temperature rise due to ultrasound absorption at a bone/soft-tissue interface, The Journal of the Acoustical Society of America 115(6): 2887.

Napoli, A., Anzidei, M., Marincola, B., Brachetti, G., Ciolina, F., Cartocci, G., Marsecano, C., Zaccagna, F., Marchetti, L., Cortesi, E. \& Catalano, C. (2013a). Primary Pain Palliation and Local Tumor Control in Bone Metastases Treated With Magnetic Resonance-Guided Focused Ultrasound, Investigative Radiology 48(6): 351-8.

Napoli, A., Anzidei, M., Marincola, B. C., Brachetti, G., Noce, V., Boni, F., Bertaccini, L., Passariello, R. \& Catalano, C. (2013b). Mr imaginge guided focused ultrasound for treatment of bone metastasis, RadioGraphics 33(6): 1555-1568.

Nell, D. \& Meyers, M. (2010). Thermal effects generated by high-intensity focused ultrasound beams at normal incidence to a bone surface, The Journal of the Acoustical Society of America 127(1): 549-559.

Partanen, A., Mougenot, C. \& Vaara, T. (2009). Feasibility of agar-silica phantoms in quality assurance of mrghifu., AIP Conference Proceedings 1113(1): 296 - 300.

Pennes, H. (1948). Analysis of tissue and arterial tem-peratures in the resting human forearm, Journal of Applied Physiology 1: 93-122.

Pinton, G., Aubry, J., Bossy, E., Muller, M., Pernot, M. \& Tanter, M. (2012). Attenuation, scattering, and absorption of ultrasound in the skull bone, Med. Physics 39(1): 299-307.

Pinton, G., Aubry, J., Fink, M. \& Tanter, M. (2011). Effects of nonlinear ultrasound propagation on high intensity brain therapy, Med. Physics 38(3): 1207-1216.

Ramsay, E., Mougenot, C., Kazem, M., Laetsch, T. W. \& Chopra, R. (2014). Temperature-dependent MR signals in cortical bone: Potential for monitoring temperature changes during high-intensity focused ultrasound treatment in bone, Magn Reson Med . doi: 10.1002/mrm.25492.

Rieke, V. \& Butts Pauly, K. (2008). MR thermometry, J. Magn. Reson. Imaging 27(2): 376-390.

Scott, S., Prakash, P., Salgaonkar, V., Jones, P., Cam, R., Han, M., Rieke, V., Burdette, E. \& Diederich, C. (2013a). Interstitial ultrasound ablation of tumors within or adjacent to bone: Contributions of preferential heating at the bone surface, Proc. SPIE 8584, Energy-based Treatment of Tissue and Assessment VII, pp. 85840Z-12.

Scott, S., Prakash, P., Salgaonkar, V., Jones, P., Cam, R., Han, M., Rieke, V., Burdette, E. \& Diederich, C. (2013b). Approaches for modelling interstitial ultrasound ablation of tumours within or adjacent to bone: Theoretical and experimental evaluations, Int. J. Hyperthermia 29(7): 629642 . 
Scott, S., Salgaonkar, V., Prakash, P., Burdette, E. \& Diederich, C. (2014). Interstitial ultrasound ablation of vertebral and paraspinal tumours: Parametric and patient-specific simulations, Int. J. Hyperthermia 30(4): 228-244.

Weilbaecher, K. N., Guise, T. A. \& McCauley, L. K. (2011). Cancer to bone: a fatal attraction, Nat Rev Cancer 11(6): 411-425. 